\title{
Histoplasmosis diseminada progresiva en una cohorte de pacientes coinfectados con el VIH
}

\author{
Progressive disseminated histoplasmosis in a \\ cohort of patients with HIV coinfection
}

\author{
Ángela M. Tobón, Alejandra Medina, luisa Orozco, Carlos Restrepo, \\ Diego Molina, Catalina de Bedout, Ángela Restrepo • Medellín (Colombia)
}

\section{Resumen}

Objetivo: identificar manifestaciones clínicas y métodos de laboratorio conducentes al diagnóstico de histoplasmosis diseminada progresiva (HDP), en una cohorte de pacientes coinfectados con el VIH.

Diseño: análisis retrospectivo de historias clínicas. No intervención adicional.

Pacientes: cuarenta pacientes con HDP e infección por VIH del Hospital La María, enero de 1992 a diciembre de 2008.

Mediciones: datos demográficos, signos, síntomas y exámenes de laboratorio que permitieron el diagnóstico de HDP.

Resultados: cuarenta pacientes, 34 hombres (85\%), y seis mujeres (15\%), con edades promedio de 33.4 y 27 años, respectivamente.

En éstos predominaron: tos $(77.5 \%)$, fiebre $(90 \%)$ y anorexia con pérdida de peso en $92.5 \%$ y $77.4 \%$, respectivamente. Lesiones en piel en $55 \%$ y en mucosa en $50 \%$, crecimiento ganglionar en $62.5 \%$ y hepatomegalia en $52.5 \%$. Menos frecuentes fueron disnea, esplenomegalia, vómito, diarrea y cefalea. Presentaron anemia el $85 \%$, leucopenia el $52.5 \%$ y trombocitopenia el $30 \%$ de los pacientes.

Exámenes micológicos: directo positivo en 21 muestras, de lavado broncoalveolar siete, piel seis, ganglio 12, biopsia transbronquial una y lesión de mucosa una. Se aisló H. capsulatum en todos los pacientes a partir de muestras de piel 10, ganglio 18, sangre tres, médula ósea una, lavado broncoalveolar (LBA) 10, y mucosa tres. Además, en siete pacientes, el hongo se aisló de más de un sitio anatómico. La serología realizada en 13 pacientes, se mostró reactiva en la inmunodifusión en gel de agar (IDGA) en 11 y en la fijación del complemento (FC) en 10.

Conclusión: ante un cuadro clínico compatible con HDP en paciente con infección por VIH que presenta fiebre, pérdida del estado general, crecimiento ganglionar, compromiso medular y piel y mucosas, el laboratorio permitirá confirmar fácilmente el diagnóstico de la sospecha clínica de la entidad (Acta Med Colomb 2011; 36: 63-67).

Palabras clave: histoplasmosis diseminada progresiva, infección por VIH, aspectos clínicos, diagnóstico por laboratorio.

\footnotetext{
Abstract

Objective: to identify the clinical manifestations and laboratory methods leading to the diagnosis of progressive disseminated histoplasmosis (PDH) in a cohort of patients co-infected with HIV.

Design: retrospective analysis of case histories. No further intervention.

Patients: forty patients with PDH and HIV infection from Hospital La María. January 1992 to December 2008.

Measurements: demographic data, signs and symptoms, and laboratory tests leading to the diagnosis of PDH.

Results: 40 patients, 34 males (85\%) and 6 females (15\%), with mean ages of 33.4 and 27 years, respectively.

The dominant symptoms were: cough (77.5\%), fever (90\%), and anorexia with weight loss in $92.5 \%$ and $77.4 \%$, respectively. Skin lesions in 55\% and mucosal lesions in 50\%, enlargement of lymph nodes in $62.5 \%$, and hepatomegaly in $52.5 \%$. Less frequent manifestations were dyspnea, splenomegaly, vomit-
}

Dra. Ángela M. Tobón: Internista, Unidad Micología Médica y Experimental, Corporación para Investigaciones Biológicas (CIB); Dras. Alejandra Medina y Luisa Orozco: Hospital La María; Dres. Carlos Restrepo y Diego Molina: Internistas, Unidad Micología Médica y Experimental, CIB; Lic. Catalina de Bedout: Bacteriología; Dra. Ángela Restrepo: MSc, Ph.D. Unidad Micología Médica y Experimental, CIB. Medellín (Colombia).

Correspondencia: Dra. Ángela M. Tobón. CIB, Medellín.

E-mail: atobon@cib.org.co

El presente trabajo no ha recibido subvención económica, y los autores no poseen conflicto de intereses.

Recibido: 16/III/2011 Aceptado: 08/VI/2011 
ing, diarrhea, and headache. Anemia was found in $85 \%$, leucopenia in $52.5 \%$, and thrombocytopenia in $30 \%$ of the patients.

Mycological tests: directly positive in 21 samples: bronchoalveolar lavage (7), skin (6), lymph node (12), transbronchial biopsy (1), and mucosal lesion (1). H. capsulatum was isolated in all patients from samples of: skin (10), lymph node (18), blood (3), bone marrow (1), bronchoalveolar lavage fluid (10), and mucosa (3). Moreover, the fungus was isolated in more than one anatomical site in 7 patients. Serology carried out in 13 patients was reactive on agar gel immunodifusion test in 11 cases and on complement fixation in 10 cases.

Conclusion: in the face of a clinical picture consistent with PDH in a patient with HIV who presents with fever, constitutional symptoms, enlargement of lymph nodes, and involvement of the bone marrow, skin, and mucous membranes, laboratory testing allows easy confirmation of the clinically suspected condition (Acta Med Colomb 2011; 36: 63-67).

Key-words: progressive disseminated histoplasmosis, HIV infection, clinical aspects, laboratory diagnosis.

\section{Introducción}

La histoplasmosis es una micosis sistémica, endémica, de zonas templadas o tropicales, causada por la inhalación de las estructuras infectantes (microconidias) del hongo dimórfico Histoplasma capsulatum (1). La infección por éste es común en Norte América alrededor de los ríos Ohio y Mississippi, así como en diversas regiones de Centro América y Sur América (2), y está asociada con actividades laborales o recreacionales que ocasionan disturbios del suelo, donde se encuentra material contaminado con el hongo.

A pesar del incremento en el uso de la terapia antirretroviral altamente activa (HAART), la histoplasmosis se mantiene como una infección oportunista importante entre los pacientes infectados con VIH, los cuales desarrollan con mayor frecuencia una enfermedad diseminada progresiva (HDP), definida por compromiso extrapulmonar clínicamente manifiesto debido a la multiplicación de $H$. capsulatum en múltiples órganos principalmente en hígado, bazo, ganglios, médula ósea y piel, diseminación que el paciente es incapaz de controlar debido a su deficiencia inmune $(1,2)$. La incidencia de la histoplasmosis dependerá de la exposición previa al hongo en áreas endémicas para la infección, la que podrá reactivarse posteriormente cuando el individuo adquiera una condición de inmunosupresión. La entidad puede corresponder a la primera manifestación de infección por VIH en un $26.1 \%$ de los casos y a medida que disminuye la función inmune en los pacientes, aumenta el riesgo tanto para desarrollar la enfermedad como para el grado de severidad (3). En este grupo de pacientes, la enfermedad se presenta en estadios avanzados de la inmunosupresión, generalmente con CD4 $<150$ células /mL y sin tratamiento tiene un curso rapidamente fatal (4). Muchos factores pueden contribuir a este pobre desenlace, entre los cuales se encuentra la demora en establecer el diagnóstico, atribuido probablemente a síntomas inespecíficos de la enfermedad, falta de reconocimiento de la entidad fúngica y retraso en la atención médica (4).

En Colombia, de acuerdo con la encuesta nacional sobre histoplasmosis realizada por el Instituto Nacional de Salud (INS) y la Corporación para Investigaciones Biológicas
(CIB) entre los años 1992 y 2008, se analizaron 434 reportes de pacientes con histoplasmosis confirmada, $70.5 \%$ correspondió a HDP en pacientes con SIDA y $7 \%$ en pacientes con otro estado de inmunosupresión. Así mismo, en $29.3 \%$ de estos pacientes, la HDP, constituyó la enfermedad definitoria de SIDA (5).

Los síntomas de la HDP incluyen fiebre, malestar general, anorexia y pérdida de peso, acompañada por pancitopenia y con hallazgo al examen físico de hepatoesplenomegalia, linfadenopatía, palidez, petequias, y en algunos pacientes lesiones en piel y mucosas $(2,6,7)$.

A pesar de la disponibilidad de terapia antirretroviral en todos los pacientes infectados con el VIH, y la efectividad de la terapia antifúngica, la HDP cursa con una mortalidad entre 20 y $40 \%$ en los diferentes estudios $(8,9)$.

Dada la importancia del diagnóstico oportuno de la entidad, en este trabajo se analizaron las características clínicas, epidemiológicas, y los métodos de laboratorio que condujeron al diagnóstico de HDP en 40 pacientes con SIDA.

\section{Pacientes y métodos}

Se realizó un estudio retrospectivo, que incluyó la revisión de historias clínicas de 40 pacientes con diagnóstico confirmado de HDP y coinfección con VIH, hospitalizados durante el periodo enero de 1992 a diciembre de 2008, en el Hospital La María, entidad pública, que atiende los pacientes del régimen subsidiado en salud del departamento de Antioquia, Colombia. Se excluyeron aquellos pacientes con otra infección oportunista simultánea con la histoplasmosis en un esfuerzo para caracterizar más precisamente el cuadro clínico correspondiente a la HDP en pacientes infectados con VIH, sin interferencia con signos y síntomas que pudieran ser debidos a otra infección oportunista concomitante. Se determinaron las características demográficas, clínicas y los métodos de laboratorio utilizados para el diagnóstico de la enfermedad fúngica.

El diagnóstico de histoplasmosis se estableció por la identificación del Histoplasma capsulatum en exámenes directos por tinciones de Wright y plata metenamina, por 
histopatología con tinciones de PAS y plata metenamina, aislamiento en cultivo y serología

Se realizó la detección de anticuerpos contra Histoplas$m a$ por inmunodifusión en gel de agar (IDGA) con histoplasmina y fijación del complemento (FC) con antígenos de levadura y con histoplasmina $(2,7)$.

Se definió HDP, por el compromiso de algún órgano extrapulmonar y, a su vez se confirmó la infección por VIH, mediante la prueba de ensayo inmunoenzimático (ELISA) para VIH y confirmado por la técnica de Western Blot.

Entre los datos demográficos se incluyeron edad, género, lugar de residencia, ocupación y administración de HAART al momento del diagnóstico de HDP. Además signos y síntomas presentes en los pacientes y exámenes de laboratorio que permitieron el diagnóstico de HDP.

La caracterización del estado inmune del paciente, se estableció por medio del recuento de linfocitos TCD4, en aquellos que contaron con este examen.

\section{Análisis estadístico}

Los datos obtenidos de la revisión de historias clínicas fueron almacenados en una base de datos en formato Microsoft Excel 2007@, para el análisis de éstos, se obtuvo las frecuencias absolutas y relativas de cada una de las variables de interés.

\section{Resultados}

De la revisión de las historias hechas en el transcurso de 16 años, se encontraron 40 pacientes con diagnóstico de coinfección VIH e histoplasmosis, siendo 34 hombres (85\%) y seis mujeres (15\%) con una edad promedio de 30 años, 33 para los hombres y 27 años para la mujeres.

La mayoría de los pacientes residían en Medellín al momento de los diagnósticos, y solamente recibían HAART 13 pacientes correspondiente a $32.5 \%$. El antecedente ocupacional o factor de riesgo de exposición a fuentes posiblemente contaminadas con el hongo, sólo se encontró en cinco pacientes (12.5\%) (Tabla 1).

Los principales hallazgos clínicos fueron anorexia (92.5\%), fiebre (90\%), anemia (85\%), pérdida de peso y tos $(77.5 \%)$. El crecimiento ganglionar estuvo presente en $62.5 \%$ de los pacientes, así como lesiones en piel en 55\% y en mucosas en $50 \%$ de los casos. Otros hallazgos menos frecuentes fueron hepatoesplenomegalia, disnea, diarrea, vómito y cefalea. Se llama la atención sobre la presencia de alteraciones hematológicas, con anemia en $85 \%$ de los casos, leucopenia y trombocitopenia en 52.5 y $30 \%$ respectivamente (Tabla 2).

Se realizó estudio radiológico del tórax en 37 de los 40 pacientes, encontrándose anormalidad en 27 de ellas (73\%), con infiltrados intersticiales en 15 pacientes, infiltrado miliar en siete, nodular en cuatro y mixtos (alveolar e intersticial) en un paciente.

Sólo se solicitó serología para hongos en 13 pacientes, la cual se encontró reactiva mediante la IDGA en 11 pacientes
Tabla 1. Caracterización demográfica de 40 pacientes con HDP e infección por VIH.

\begin{tabular}{|ll|}
\hline $\begin{array}{l}\text { Sexo } \\
\text { Masculino/Femenino (\%) }\end{array}$ & $34(85) / 6(15)$ \\
\hline $\begin{array}{l}\text { Edad promedio } \\
\text { Masculino/Femenino (años) }\end{array}$ & $33 / 27$ \\
\hline Residencia (N) & $\begin{array}{l}\text { Medellín (25) } \\
\text { Otros municipios (15) }\end{array}$ \\
\hline Administración HAART N (\%) & $13(32.5)$ \\
\hline Antecedente de riesgo de infección por H. capsulatum N (\%) & $5(12.5)$ \\
\hline Linfocitos T CD4 N promedio (rango) & $36.43(3$-172) \\
\hline *HDP: Histoplasmosis Diseminada Progresiva. \\
\hline
\end{tabular}

Tabla 2. Manifestaciones clínicas de 40 pacientes con HDP e infección por VIH.

\begin{tabular}{|l|c|c|}
\hline Signos y síntomas & $\mathbf{N}=\mathbf{4 0}$ & $\%$ \\
\hline Fiebre & 36 & 90 \\
Anorexia & 37 & 92.5 \\
Pérdida de peso & 31 & 77.5 \\
Tos & 31 & 77.5 \\
Crecimiento ganglionar & 25 & 62.5 \\
Hepatomegalia & 21 & 52.5 \\
Lesiones en piel & 22 & 55 \\
Lesiones en mucosas & 20 & 50 \\
Anemia & 34 & 85 \\
Leucopenia & 21 & 52.5 \\
Trombocitopenia & 12 & 30 \\
Diarrea & 13 & 47.5 \\
Disnea & 12 & 32.5 \\
Esplenomegalia & 19 & 30 \\
Vómito & 11 & 27.5 \\
Cefalea & & 35 \\
\hline
\end{tabular}

con bandas $\mathrm{M}$ y/o $\mathrm{H}$, y en 10 pacientes con títulos > 1:32 a la fijación del complemento.

La posibilidad diagnóstica de histoplasmosis se informó rápidamente, por la presencia de blastoconidias compatibles con Histoplasma capsulatum al examen directo por tinción de diferentes muestras, siendo más frecuente en tejido de biopsia ganglionar y lavado broncoalveolar (LBA), seguido por lesiones de piel y mucosas.

El aislamiento del hongo en cultivo, como prueba de laboratorio confirmatoria de la enfermedad fúngica, se realizó principalmente a partir de tejido ganglionar, en el cual de 19 muestras cultivadas se aisló en 18 de ellas (94.7\%), seguido por LBA en 11 de 19 realizados (57.89\%), y piel en 10 de $14(71.42 \%)$ y en menor frecuencia, en lesiones de mucosas tres de 10, sangre tres de cinco, esputos dos de dos y médula ósea uno de uno. No fue posible el aislamiento del hongo en las cinco muestras de líquido cefalorraquídeo que fueron cultivadas (Tabla 3). 
Tabla 3. Diagnóstico de histoplasmosis diseminada progresiva en 40 pacientes co-infectados con VIH.

\begin{tabular}{|l|c|c|}
\hline Tipo de muestra & $\begin{array}{c}\text { Directo positivo }\left(\mathbf{N}^{\mathbf{0}} \mathbf{2 1}\right) \\
\mathbf{N}(\%)\end{array}$ & $\begin{array}{c}\text { Cultivo positivo (No40) } \\
\mathbf{N}(\%)\end{array}$ \\
\hline Ganglio & $12(57.14)$ & $18(45)$ \\
LBA & $7(33.3)$ & $11(27.5)$ \\
Piel & $6(28.5)$ & $10(25)$ \\
Mucosa & $1(4.76)$ & $3(7.5)$ \\
Sangre & 0 & $3(7.5)$ \\
Esputos & $2(9.52)$ & $2(5)$ \\
Biopsia pulmonar & $1(4.76)$ & $1(2.5)$ \\
Medula ósea & 0 & $1(1.5)$ \\
Liqudo cefalorraquídeo & 0 & 0 \\
\hline
\end{tabular}

En siete pacientes (17.5\%) el aislamiento se realizó a partir de más de una muestra, especialmente ganglio y LBA simultáneamente.

Fue posible conocer el recuento de CD4 en 16 de los 40 pacientes (40\%), entre los cuales el promedio fue de 36.43 células/mL (rango 3-172).

\section{Discusión}

Al momento de la infección con Histoplasma capsulatum la gran mayoría de los individuos no presentan síntomas, o éstos son muy leves, lo cual impide reconocer la infección en la mayoría de ellos. El hongo se disemina a diferentes tejidos y el sistema inmune intacto controlará la multiplicación de éste y simultáneamente el progreso de la infección $(10,11)$. La persistencia de estructuras fúngicas latentes pero aún viables en focos aislados podrá conducir al desarrollo de enfermedad, cuando el individuo presente alguna alteración de su sistema inmune celular o cuando la carga fúngica de infección sea muy grande (2).

Este es el caso de los pacientes infectados con el VIH, en quienes la histoplasmosis continúa presentándose con frecuencia como una infección oportunista, especialmente en aquellos pacientes residentes en áreas endémicas, o en quienes han viajado o vivido previamente en éstas (9). En este grupo de pacientes es inaparente el antecedente de exposición al hongo y ausente el cuadro clínico de histoplasmosis pulmonar aguda, lo cual dificulta enormemente su diagnóstico y como consecuencia empeora el pronóstico (4). En el presente trabajo sólo cinco pacientes presentaron un antecedente de actividades de riesgo para infección por Histoplasma, dificultando la sospecha de la infección fúngica.

El cuadro clínico de HDP en pacientes con SIDA es inespecífico, pero desde las primeras publicaciones se ha informado la presencia de fiebre, pérdida de peso y tos, como hallazgos frecuentes en más de $50 \%$ de los pacientes $(12,13)$. En nuestro grupo de pacientes éstas también fueron las principales manifestaciones, con fiebre y anorexia presentes en $90 \%$ y $92 \%$ respectivamente, acompañadas por pérdida de peso y tos en $77 \%$ de los casos, lo cual fue informado por el grupo en publicaciones anteriores $(6,7)$.

El crecimiento ganglionar se presentó en $63 \%$ de los pacientes y permitió el diagnóstico temprano de la entidad en $57 \%$ y el aislamiento definitivo del hongo en $45 \%$ de los casos; similar a lo informado en otras series, entre el 41 y $48 \%$, y también como un sitio muy importante para el aislamiento del hongo $(8,14)$.

Uno de los aspectos importantes que la presente revisión quiere resaltar, es el frecuente compromiso por Histoplasma de la piel en $55 \%$ y las mucosas en $50 \%$ de nuestros pacientes, lo cual contrasta con otras publicaciones en pacientes en Alabama 6.5\% (8), Guyana Francesa $15.7 \%$ (14) y $10 \%$ como informe general en la literatura norteamericana (15), pero informado con mayor frecuencia, hasta $66 \%$ en pacientes en Sur América (9). La apariencia más común de estas lesiones son las pápulas y úlceras con costras, presentes en diferente estadío de evolución y también la presencia de úlceras dolorosas en cavidad oral, principalmente dorso de la lengua. La importancia de la presencia de este compromiso mucocutáneo radica en el hecho de que asociadas al cuadro clínico general, conducen a la sospecha diagnóstica de HDP y permiten de una manera rápida y poco invasiva, la visualización y aislamiento del hongo por raspado o biopsia.

El 73\% de los estudios radiológicos del tórax evidenciaron compromiso por infiltrado intersticial reticulonodular difuso con mayor frecuencia, lo cual concuerda con lo informado en la literatura, alrededor del 70\%. (16.17). Este tipo de compromiso es explicado por el ingreso respiratorio del hongo al organismo y permitió, en nuestro caso, al aislamiento de éste a través del LBA, en una cuarta parte de los pacientes.

Las dificultades para tener acceso a los servicios del hematólogo, impidieron mayores estudios fúngicos en médula ósea, compromiso sospechable por la alta frecuencia de anemia, leucopenia y trombocitopenia en 85,53 y $30 \%$ de los pacientes, respectivamente. Este compromiso puede, igualmente, ser secundario a la infección por el VIH o medicamentos que recibe el paciente, por lo cual debe insistirse en el estudio de médula ósea, ya que se ha informado el aislamiento del hongo en $69 \%$ de los casos en aspirado o biopsia de este tejido (12).

El VIH induce disfunción de las células B y de los linfocitos T CD4, lo cual explica la pobre respuesta a través de los anticuerpos contra H. capsulatum, por lo cual aun en presencia de infección activa, la serología suele ser negativa en más del $50 \%$ de los pacientes inmunocomprometidos especialmente por VIH, con mayor probabilidad de ser positiva en las formas más crónicas de la presentación clínica de la enfermedad, y menor en las formas agudas con menos de 15 días de evolución de la sintomatología $(2,18,19)$. Este concepto explica el porqué sólo se solicitó esta prueba serológica en 13 pacientes; no obstante, llama la atención que tal prueba fue positiva en 11 de los 13 pacientes a través de la IDGA y en 10 pacientes en la FC con títulos $\geq 1: 32$. 
En resumen, en pacientes con infección por el VIH, el cuadro clínico de fiebre, pérdida de peso y tos, acompañado por crecimiento ganglionar, lesiones mucocutáneas y presencia de bicitopenia o pancitopenia por pruebas de laboratorio, debe hacer pensar en el diagnóstico de histoplasmosis diseminada progresiva. Una vez exista esta sospecha, el laboratorio establecerá fácilmente el diagnóstico definitivo a través del estudio de los diferentes tejidos comprometidos.

Es necesario establecer el diagnóstico oportuno de la HDP, para mejorar el resultado de la terapia antimicótica en pacientes con alteración inmune por la infección con el VIH.

\section{Referencias}

1. Corti ME, Negroni R, Esquivel P, Villafañe MF. Histoplasmosis diseminada en pacientes con SIDA: análisis epidemiológico, clínico, microbiológico e inmunológico de 26 pacientes. Enf Emerg 2004; 6: 8-15.

2. Kauffman CA. Histoplasmosis: a Clinical and Laboratory Update. Clin Microbiol Rev 2007; 20: 115-32.

3. Hajjeh RA, Pappas PG, Henderson H, Lancaster D, Bambergci DM, Skahan KJ et al. Multicenter case control study of risk factors for histoplasmosis in HIVinfected persons. Clin infect dis 2001; 32: 1215-20.

4. Couppié P, Sobesky M, Aznar C, Bichat S, Clyti E, Bissuel F et al. Histoplasmosis and Acquired Innmunodeficiency syndrome. A study of prognostic factors. Clin infect dis 2004; 38: 134-8.

5. Arango M, Castañeda E, De Bedout C, Agudelo C, Agudelo CI, Tobón A, et al. Histoplasmosis: resultado de la encuesta nacional 1.992-2008. Infectio 2010; 14.

6. Tobón AM, Agudelo CA, Rosero DS, Ochoa JE, De Bedout C, Zuluaga A et al. Disseminated Histoplasmosis: a comparative study between patients with acquiried immunodeficiency syndrome and non-human immunodeficiency virus-infected individuals. Am J Trop Hyg 2005; 73: 576-82.

7. Tobón AM, Franco L, Correa AL, Bedoya F, Ortega J, Soto M, et al . La
Histoplasmosis en el adulto. Bases para su diagnóstico. Act Med Colomb 1997; 22(6) 277-84.

8. Baddley JW, Sankara IR, Rodriguez JM, Pappas PG, Many Jr WJ. Histoplasmosis in HIV-infected patients in a southern regional medical center: poor prognosis in the era of highly active antiretroviral therapy. Diag Microbiol and Infec Dis 2008; 62: 151-6.

9. Deepe, Jr. G. Histoplasma capsulatum. Mandel GL, Bennett JE, Dolin R. Principles and practice of Infectious Diseases. Seventh Edition. Philadelphia. Churchill Livingstone. Elsevier. 2010; 3305-18.

10. Wheat LJ, Slama TG, Norton JA. Risk factors for disseminated or fatal histoplasmosis. Ann Intern Med 1982; 96:159-63.

11. Goodwin RA, Loyd Jr JE, and des Prez RM. 1981. Histoplasmosis in norma hosts. Medicine (Baltimore) 1981; 60: 231-66.

12. Johnson PC, Khardori N, Najjar AF, Butt F, Mansell PWA, Sarosi G. Progressive Disseminated Histoplasmosis in patients with acquired Immunodeficiency syndrome. Am J Med 1988; 85: 152-8.

13. Wheat LJ, Connolly-Stringfield PA, Baker RL, Curfman MF, Eads ME, Israel KS, et al. Disseminated Histoplasmosis in the aquired immune deficiency syndrome: clinical findings, diagnosis and treatment, and review of the literature. Medicine (Baltimore) 1990; 69: 244-60.

14. Nacher M, Sarazin F, El Guedj M, Vaz T, Alvarez F, Nasser V, Randrianjohany A, et al. Increased Incidence of Disseminated Histoplasmosis following highly active antiretroviral therapy initiation. J Aquir Immune Defic Syndr 2006; 41: 468-70.

15. Eidbo J, Sanchez RI, Tschen JA. Cutaneous manifestation of histoplasmosis in the aquired immune deficiency syndrome. Am J Surg Pathol 1993; 17: 110-6.

16. Conces DJ Jr, Stockberger SM, Tarver RD, Wheat LJ. Disseminated Histoplasmosis in AIDS: findings on chest radiographs. AM J Roentgenol 1993; 160: 15-19.

17. Hage Ch A, Wheat LJ, Loyd J, Allen SD, Blue D, Knox KS. Pulmonary Histoplasmosis. Seminars in Respir and Crit Care Med 2008; 29: 151-65.

18. Wheat LJ. Laboratory diagnosis of histoplasmosis: update. Semin Respir Infect 2000; 16: 131-40.

19. Tobón AM, Agudelo CA. Micosis sistémicas. En: Infectología: Métodos diagnósticos en Medicina Clínica. Enfoque práctico. Murgueitio R, Prada G, Archila PE. Celsus. ACMI. Colombia 2007. 